\title{
Performance Analysis for Clusters of BLE Devices with Heterogeneous Parameters
}

\author{
Gaoyang Shan, Seung-hun Shin, and Byeong-hee Roh
}

\begin{abstract}
As one of the most promising technologies, Bluetooth low energy has been widely utilized for supporting various Internet of Things services and applications. To create connection between BLE devices, neighbor discovery process is an essential procedure. However, because of the effect of signal collision problem between BLE devices, the neighbor discovery performance is degraded seriously. In this paper, we consider clustering BLE devices, where the devices in same cluster own same parameter settings. We analyze the signal collision probability, and the discovery delay of these clustering devices during the discovery process by simulator. The simulation result shows the relationship between discovery performance and the rate of the number devices in each cluster.
\end{abstract}

Index Terms-Bluetooth low energy (BLE), neighbor discovery process, internet of things (IoT), heterogeneous network parameters.

\section{INTRODUCTION}

With the development of various Internet of things (IoT) services and applications, a growing number of objects are going to be connected with internet [1]. To support these services and applications, many communication technologies are studied for the utilization in IoT, such as ZigBee, radio frequency identifiers (RFIDs), Bluetooth, and Bluetooth low energy (BLE), and so on [2]. Each technology owns their distinct features. BLE has been utilized in many areas because of its low-power solution and suitable transmission range. The examples of BLE utilization areas in IoT include indoor localization [3], [4], healthcare [5], [6], smart home [7], [8], and so on.

In BLE network, to create connection between BLE devices (master BLE device and slave BLE device), the master BLE device (scanner) should discover the slave device (advertiser) firstly. The advertiser sends advertisement packet on three channels periodically, and can be discovered by scanner when an advertisement packet was received by the scanner. Due to only three channels are used for sending advertisement packet, signal collision is serious when large number of advertisers are co-exist in a narrow area. This impacts the discovery delay significantly, and then increases the energy consumption of BLE device, and reduce the life time of BLE devices.

Many existing literatures discussed the effect of signal collision on discovery delay and energy consumption. In [9],

Manuscript received September 4, 2018; revised November 12, 2018.

Gaoyang Shan and Byeong-hee Roh are with the Dept. of Computer Engineering, Ajou University, Suwon, 16499, Korea (e-mail: shanyang166@ajou.ac.kr, bhroh@ajou.ac.kr).

Seung-hun Shin is with Dasan University College, Ajou University, Suwon 16499, Korea (e-mail: sihnsh@ajou.ac.kr). the author use a pure ALOHA system model to analyze the discovery delay for a tagged advertiser. The tradeoff between discovery delay and energy consumption of a tagged advertiser has been analyzed in [10]. The analytical for analyzing the time for discovering all existing advertisers in surround has been derived in [11]. In [12] and [13], the authors derived their model to analyze the energy consumption of advertiser during the discovery process. The analysis of these literatures shows us, the discovery performance could be improved according the adjustment of BLE parameters. The longer advertisement interval could reduce the signal collision probability effectively. An optimal parameter setting algorithm is proposed in [14]. However, all these studies only consider the same parameter settings for each advertiser. In practice, each BLE device may set different parameters, it is difficult to make each advertiser set same parameter.

In this paper, we analyze the signal collision and discovery delay with the consideration of multiple advertiser clusters. In a cluster, the advertisers own same parameter settings, and the advertiser in different cluster have different parameter settings. We analyze the relationship among the signal collision probability, discovery delay and the rate of the number of advertisers in each cluster. The main contribution of this paper is listed as follows:

- The relationship between signal collision probabilities with the rate of the number of advertisers in each cluster is shown.

- The scanning time for discovering all advertisers are shown by varying the number of advertisers in each cluster.

The rest of this paper is organized by follows: the basic BLE discovery process and advertisement packet format are briefly described in Section II. In Section III, the system model is described. The simulation results for signal collision probability and discovery delay are shown in Section IV. At last, the paper is concluded in Section V.

\section{BLE DISCOVERY PROCESS}

In BLE discovery process, two kinds of device are present: advertiser and scanner. Scanner should scan on three channel, and advertiser should send advertisement packet on three channels. The advertiser can be discovered when the packet from advertiser is received by scanner. Two kinds of scanning types are present: passive and active. In passive scanning, the scanner does not send any packet back to the advertiser. In active scanning, the scanner can send a packet for requesting more information to advertiser 
or send a connection request. Three kinds of advertising mode are present: connectable mode, scannable mode, and non-connectable and non-connectable mode. In connectable mode, the advertiser allows scanner send connection request to the advertiser and ignores the scanning request. In scannbale mode, the advertiser allows scanner send scanning request to the advertiser, and ignores the connection request. In non-connectable and non-scannable mode, the advertiser does receive any scanning request or connection request. In this section, we describe the BLE scanning process in passive scanning type and BLE advertising process in non-connectable and non-scannable advertising mode [15].

\section{A. BLE Advertising Process}

As shown in Fig. 1 (a), the advertiser generates advertisement event (AdvEvent), periodically. The period of $A d v E v e n t$ consists of two parameters: advertisement interval (AdvInterval) and advertisement delay (AdvDelay). AdvInterval is a fixed value in the range of $20 \mathrm{~ms}$ to $10.24 \mathrm{~s}$. For each AdvEvent, their AdvInterval should be same. As the definition in BLE specification [15], the AdvInterval should be an integer multiple of $0.625 \mathrm{~ms}$. AdvDelay is a random value between 0 and $10 \mathrm{~ms}$. The AdvDelay for each $A d v E v e n t$ may be different. In each AdvEvent, at most three advertising channels (channel index is 37, 38, and 39) are used for transmitting the advertisement packet ADV_IND, orderly. Also, the time interval between two neighboring ADV_INDs in an AdvEvent should be less than or equal to $10 \mathrm{~ms}$. The advertiser can be discovered when an ADV_IND was received by scanner successfully.

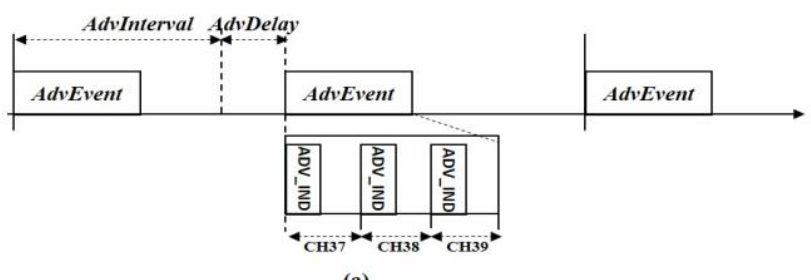

(a)

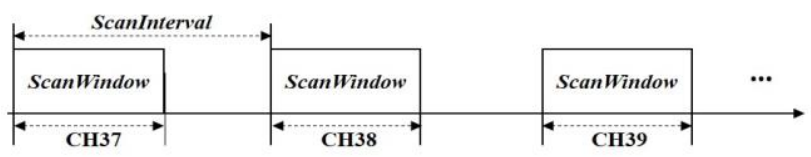

(b)

Fig. 1. BLE discovery process: (a). BLE advertising process, (b). BLE scanning process.

\section{B. BLE Scanning Process}

In the other side, the scanner scans on three channels (channel index is 37, 38, and 39) orderly. As shown in Fig. 1 (b), the scanning operation is performed in each scanning window (ScanWindow). The scanning period is defined as ScanInterval. The remaining time in each ScanInterval is sleeping time. ScanWindow should be less than or equal to ScanInterval. The ScanInterval should be less than $10.24 \mathrm{~s}$ [15]. When the ScanWindow is same with the ScanInterval, then there is no sleeping time, and the scanner should keep scanning on each channel.

\section{The Packet Format of ADV_IND}

The packet format of ADV_IND is shown in Fig. 2.
ADV_IND consists of preamble, access address, header, advertising address $(A d v A)$, advertising data (AdvData), and CRC. The size of AdvData is 31 bytes at most. So, the packet size of ADV_IND can be calculated as 47 bytes if we consider the biggest size of AdvData.

\begin{tabular}{|c|c|c|c|c|c|}
\hline $\begin{array}{c}\text { Preamble } \\
(\mathbf{1} \text { byte })\end{array}$ & $\begin{array}{c}\text { Access address } \\
\text { ( } \text { bytes })\end{array}$ & $\begin{array}{c}\text { Header } \\
(2 \text { bytes })\end{array}$ & $\begin{array}{c}\text { AdvA } \\
(\mathbf{6} \text { bytes })\end{array}$ & $\begin{array}{c}\text { AdvData } \\
(\mathbf{0} \sim \mathbf{3 1} \text { bytes })\end{array}$ & $\begin{array}{c}\text { CRC } \\
(\mathbf{3} \text { bytes })\end{array}$ \\
Fig. 2. Packet format of ADV_IND.
\end{tabular}

\section{SYSTEM MODEL}

In this paper, we consider $N$ advertisers and one scanner coexist in a narrow area. Non-connectable and nonscannbale advertising mode is considered for each advertiser. Passive scanning type is considered for the scanner. The $\mathrm{N}$ advertisers are separated in different cluster. In the same cluster, each advertiser sets same parameters. The advertisers in different clusters set different parameters. In this paper, we consider the case of two cluster. Also, continuous scanning is considered, which means the ScanWindow is equal to the ScanInterval.
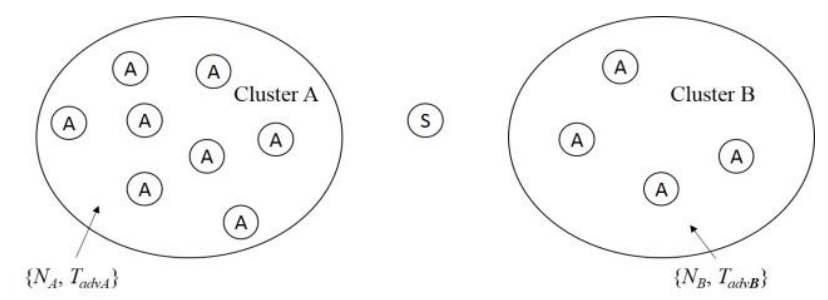

(A): Advertiser (S): Scanner

Fig. 3. Cluster A and cluster B.

Fig. 3 shows the example of two clusters: cluster A and cluster B. There are $N_{A}$ advertisers in cluster $\mathrm{A}$, and $N_{B}$ advertisers in cluster B $\left(N_{A}+N_{B}=N\right)$. In each cluster, at least one advertiser should be existing. The ScanInterval and ScanWindow of advertisers in each cluster are same. The AdvInterval of advertisers in cluster $\mathrm{A}$ is noted as $T_{a d v A}$, and the AdvInterval of advertisers in cluster $\mathrm{B}$ is noted as $T_{a d v B}$. The transmission time for a packet ADV_IND is $T_{p}$. The average AdvDelay is $T_{d}$. The signal from an advertiser may collide with the signals from cluster $\mathrm{A}$ and $\mathrm{B}$. The signal collision would occur when an advertiser starts sending packet on same channel with the tagged advertiser during the collision region, as shown in Fig. 4. If an advertiser in cluster $\mathrm{A}$ send an ADV_IND, let $p_{A A}$ the probability of this packet collides with another ADV_IND send by the advertiser in cluster $\mathrm{A}$ is

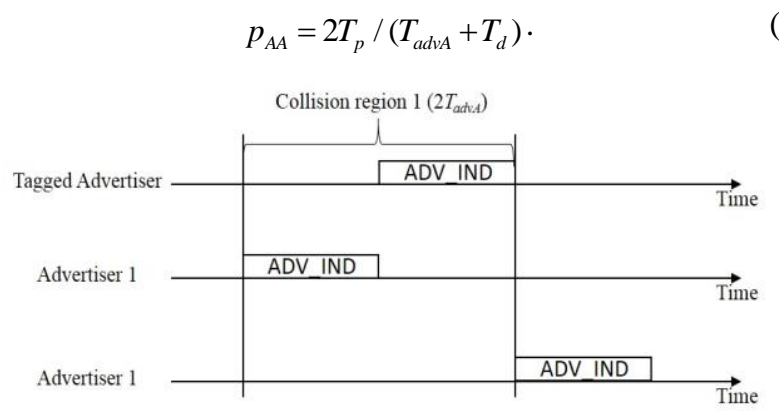

Fig. 4. Signal collision region. 
The packet send by an advertiser from cluster A also could collides with the signal send by an advertiser from cluster B. Let $P_{A B}$ be the probability of a packet send by an advertiser from cluster A collides with another packet send by an advertiser from cluster B. Then we can have

$$
p_{A B}=2 T_{p} /\left(T_{a d v B}+T_{d}\right) .
$$

By same way, we could get the probability of a packet send by an advertiser from cluster B collides with another packet send by an advertiser from cluster $\mathrm{B}, p_{B B}$, and the probability of a packet send by an advertiser from cluster $B$ collides with another packet send by an advertiser from cluster $\mathrm{A}, p_{B A}$, as

$$
\begin{aligned}
& p_{B B}=2 T_{p} /\left(T_{a d v B}+T_{d}\right) . \\
& p_{B A}=2 T_{p} /\left(T_{a d v A}+T_{d}\right) .
\end{aligned}
$$

Equation (1), (2), (3), and (4) only shows the probability of an ADV_IND collides with another one. In large number of advertisers' environment, multiple signals may collides with the ADV_IND. With the increasing of the number of advertisers, signal collision probability also increases. This would degrade the discovery performance, extend the discovery delay, and waste the energy. To evaluate the overall collision probability and discovery performance, we use simulator to test the relationship between the discovery delay and the rate of the number of advertisers in each cluster.

\section{SIMULATION RESUlTS}

A simulator is developed for the simulation [16]. In the simulation, we consider $N$ number of advertisers and one scanner. The $N$ advertisers are separated in two cluster, Cluster A and Cluster B. The ScanWindow and ScanInterval of each advertiser are considered as $10.24 \mathrm{~s}$. The AdvInterval of advertisers in cluster $\mathrm{A}$ is different with it in cluster B. in cluster A, the AdvInterval is $50 \mathrm{~ms}$, and in cluster $\mathrm{B}$, the AdvInterval is $200 \mathrm{~ms}$. The AdvDelay is a random value between 0 and $10 \mathrm{~ms}$. The parameter settings are shown in Table I.

TABLE I: PARAMETER SETTINGS

\begin{tabular}{lc}
\multicolumn{2}{c}{ TABLE I: PARAMETER SETTINGS } \\
\hline \hline Parameters & Value \\
\hline ScanInterval & $10.24 \mathrm{~s}$ \\
ScanWIndow & $10.24 \mathrm{~s}$ \\
AdvInterval (Cluster A) & $50 \mathrm{~ms}$ \\
AdvInterval (Cluster B) & $200 \mathrm{~ms}$ \\
AdvDelay & {$[0,10 \mathrm{~ms}]$} \\
\hline \hline
\end{tabular}

At first, we test the overall signal collision probability, where the overall signal collision probability is defined as the rate of the number of all lost signals caused by collision to all the number of send signals from cluster $\mathrm{A}$ and $\mathrm{B}$. The simulation results are obtained from 100 times' test. The average values are shown in each figure. We consider the total number of advertisers is 100 and 200.As shown in Fig. 5 (a), the AdvInterval of advertisers in cluster is shorter than that in cluster. $N \_A$ is the percentage of the number of advertisers in cluster $\mathrm{A}$, which is defined as

$$
N_{-} A=N_{A} / N \text {. }
$$

So, with the increasing of the number of advertisers in cluster A, the signal collision becomes serious. This is because the AdvInterval of advertisers in Cluster $\mathrm{A}$ is shorter than that in cluster B. So, when the number of advertisers in cluster $\mathrm{A}$ increases, the number of signals send by advertisers during an unite time also increases, which increases the signal collision rates. Also, for same $N \_A$, large number of advertisers leads high signal collision probability.

Signal collision affects the discovery delay directly. To explore the effect of signal collision probability on the discovery delay performance, we also do some simulation to test the discovery delay with different N_A. Fig. 5 (b) shows us the scanning time for discovering all advertisers which includes the advertiser in cluster A and cluster B. We can see the scanning time increases when the percentage of the number of advertisers in cluster A increases a bit. However, when almost all the advertisers is belong to cluster A, the scanning time is reduced. This is because when the AdvInterval of all advertisers is $50 \mathrm{~ms}$, the waiting time for each advertiser is shorter than that when the AdvInterval is $200 \mathrm{~ms}$. Except the signal collision, the waiting time for an ADV_IND also affects the scanning time for discovering all advertisers. Also, the scanning time for discovering 200 advertisers is more than the scanning time for discovering 100 advertisers. When the number of advertisers is 200, the scanning time for N_A=1 is almost two times of the scanning time for $\mathrm{N}_{-} \mathrm{A}=0$. This is because the AdvInterval of advertiser in cluster $\mathrm{B}$ is longer than that in cluster $\mathrm{B}$, and longer AdvInterval could increase the signal density, and mitigate the signal collision effectively. Low signal collision probability could improve the discovery delay performance when the number of advertisers is very large in a narrow area.

Fig. 6 shows us the scanning time for discovering 200 advertisers with different parameter settings. The blue line shows us the scanning time when $T_{a d v A}=100 \mathrm{~ms}$, $T_{a d v B}=400 \mathrm{~ms}$, and the green line shows us the scanning time when $T_{a d v A}=50 \mathrm{~ms}, T_{a d v B}=200 \mathrm{~ms}$. For same number of advertisers in each cluster, longer AdvInterval could reduce the signal collision probability, and improve the scanning performance when the number of advertisers is large. Also, when all advertisers belong to cluster $\mathrm{B}$, the scanning time when $T_{a d v A}=100 \mathrm{~ms}, T_{a d v B}=400 \mathrm{~ms}$ is longer than that when $T_{a d v A}=50 \mathrm{~ms}, T_{a d v B}=200 \mathrm{~ms}$. This is because the signal collision is not serious in this case, and the waiting time for an ADV_IND affects the scanning performance more. When $T_{a d v A}$ is very short, and $T_{a d v B}$ is very long, the scanning for discovering all advertisers is extended seriously, as shown in Fig. $6\left(T_{a d v A}=50 \mathrm{~ms}, T_{a d v B}=400 \mathrm{~ms}\right)$. For $N_{-} A=0.9$, the scanning time when $T_{a d v A}$ is $50 \mathrm{~ms}$ and $T_{a d v B}$ is $1 \mathrm{~s}$ is almost five times of the other two case. This is because when $N \_A=0.9$, there are 180 advertisers (in cluster A) set their AdvInterval as $50 \mathrm{~ms}$, and the other 20 (in cluster B) advertisers set their AdvInterval as $1 \mathrm{~s}$. In this case, the advertisers in cluster B get serious collision from cluster A. The AdvInterval of advertisers in cluster $\mathrm{B}$ is $1 \mathrm{~s}$. So, the expected time for receiving an ADV_IND from the 
advertiser in cluster B is very long. This extends the scanning time for discovering all advertisers, and also degrade the BLE scanning performance.

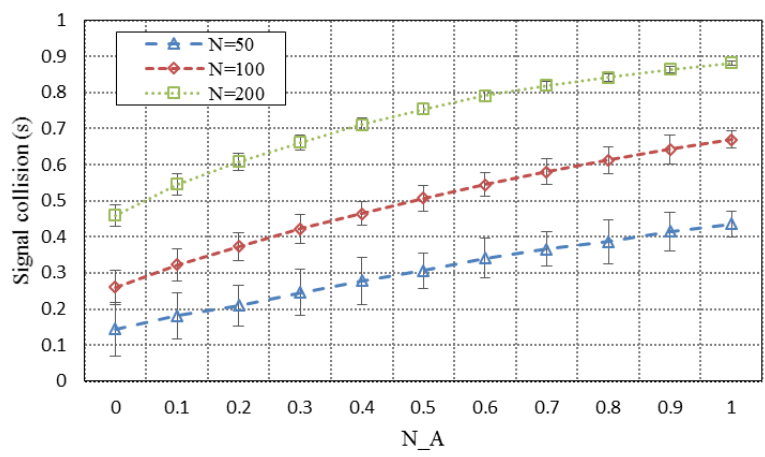

(a)

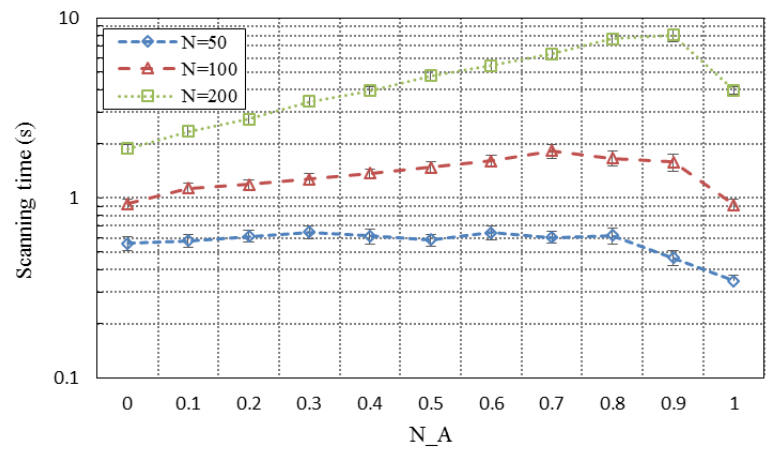

(b)

Fig. 5. (a). Collision probability, (b). Scanning time for discovering all advertisers $\left(T_{a d v A}=50 \mathrm{~ms}, T_{a d v B}=200 \mathrm{~ms}\right)$.

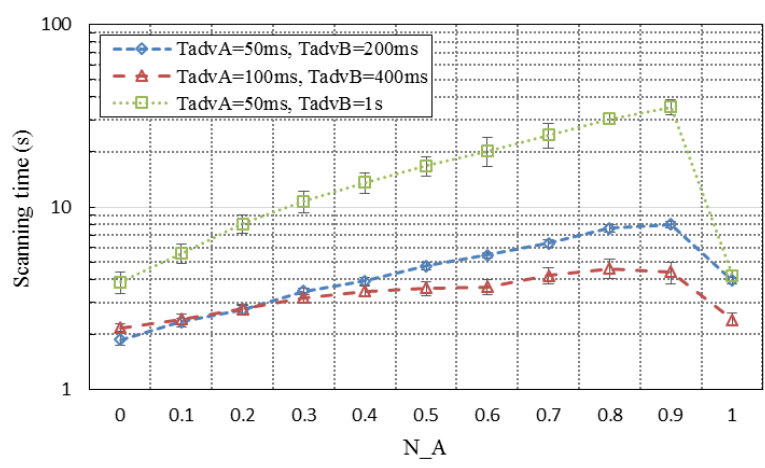

Fig. 6. Scanning time with different parameters settings.

\section{CONCLUSION}

With the development of Internet of Things (IoT) services and applications, more and more devices would be connected to internet according BLE technology. Subsequently, more serious signal collision would degrade the discovery performance during the BLE discovery process. Also, each BLE device may have different parameter settings. This cause the performance analysis complexity. In this paper, we analyzed the signal collision probability and scanning time for discovering all advertisers with the consideration of clustering BLE devices by simulator, where the BLE devices in each cluster have different parameter settings. According the simulation results we shows the effects of signal collision on the discovery performance. When major BLE advertisers set short AdvInterval, and small number of advertisers set longer AdvInterval, the discovery delay is extended. These analysis results could provide reference to BLE services providers to achieve good discovery performance.

In this paper, we has done the analysis of BLE discovery performance based on simulator with the considering of two clusters. In some special case, each BLE device may set different parameters, then the number of devices in each cluster may be one. In the future work, we would try to derive our analytical model to analyze the effects of the number of advertisers and BLE parameter settings (AdvInterval, ScanInterval, and ScanWindow), and more cluster would be considered in our model. Also, the energy consumption for different parameter settings would be derived. The trade-off between energy consumption and discovery delay would be given. The analysis results would be compared with the simulation results.

\section{ACKNOWLEDGMENT}

This work was supported by the National Research Foundation of Korea (NRF) grant funded by the Korean Government (MSIP) (no. NRF-2015R1A2A2A0100 5577)

\section{REFERENCES}

[1] J. Gubbi, R. Buyya, S. Marusic, and M. Palaniswami, "Internet of things (IoT): A vision, architectural elements, and future directions," Future Generation Computer Systems, vol. 29, no. 7, pp. 1645-1660, 2013.

[2] A. Al-Fuqaha, M. Guizani, M. Mohanmmadi, M. Aledhari, and M. Ayyash, "Internet of things: A survey on enabling technologies, protocols, and applications," IEEE Communication Surveys \& Tutorials, vol. 17, no. 4, pp. 2347-2376, 2015.

[3] T. A. Johnson and P. Seeling, "Localization using bluetooth device names," in Proc. ACM MobiHoc, USA: South Carolina, 2012.

[4] G. Shan, B. Park, S. Nam, B. Kim, B. Roh, and Y. Ko, "A 3dimensional triangulation scheme to improve the accuracy of indoor localization for IoT services," in Proc. of PACRIM, Aug. 2015.

[5] T. Zhang, J. Lu, F. Hu, and Q. Hao, "Bluetooth low energy for wearable sensor-based healthcare systems," in Proc. of Healthcare Innovation Conference (HIC), pp. 251-254, 2014.

[6] P. D. Martin, M. Rushanan, and T. Tantillo, "Applications of secure location sensing in healthcare," in Proc. of ACM BCB, Oct. 2016.

[7] M. Collotta and G. Pau, "Bluetooth for internet of things: A fuzzy approach to improve power management in smart homes," Computers \& Electrical Engineering, Elsevier, vol. 44, pp. 137-152, May 2015.

[8] R. A. Ramlee, D. H. Tang, and M. M. Ismail, "Smart home system for disabled people via wireless Bluetooth," in Proc. of ICSET, Bandung, Indonesia, Sep. 2012.

[9] J. Liu, C. Chen, Y. Ma, and Y. Xu, "Adaptive device discovery in bluetooth low energy networks," in Proc. of IEEE V TC Spring, Dresden, Germany, 2013.

[10] W. Jeon, M. Dwijaksara, and D. Jeong, "Performance analysis of neighbor discovery process in bluetooth low energy networks," IEEE Trans: V ehicular Technology, vol. 66, no. 2, pp. 1865-1871, Feb. 2017.

[11] A. H. Solana, D. P. D. Cerio, A. Valdovinos, and J. L. Valenzuela, "Proposal and evaluation of BLE discovery process based on new features of bluetooth 5.0," Sensors, pp.1-34, Aug. 2017.

[12] J. Liu, C. Chen, Y. Ma, and Y. Xu, "Energy analysis of device discovery for bluetooth low energy," in Proc. of IEEE V TC Fall, Las Vegas, NV, USA, Sep. 2013.

[13] R. Schrader, T. Ax, C. Rohrig, and C. Fuhner, "Advertising power consumption of bluetooth low energy systems," in Proc. of IDAACS - SWS, Offenburg, Germany, Sep. 2016.

[14] G. Shan, S. Im, and B. Roh, "Optimal AdvInterval for BLE scanning in different number of BLE devices environment," Computer Communications Workshops (INFOCOM WKSHPS), IEEE, 2016.

[15] Bluetooth SIG proprietary, "Specification of the bluetooth system," Covered Core Package Version, vol. 4, no. 2, 2014. 
[16] G. Shan, B. Lee, S. Shin, and B. Roh, "Design and implementation of simulator for analysis of BLE broadcast signal collision," in Proc. of ICOIN, Da Nang, Vietnam, Jan. 2017.

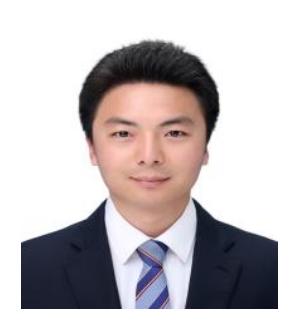

Gaoyang Shan received the B.S degree in electronics engineering from Kyonggi University, Suwon, South Korea, in 2014, and the M.S degrees in computer engineering from Ajou University, Suwon, South Korea, in 2016, respectively. $\mathrm{He}$ is currently working toward the Ph.D degree at the Department of Computer Engineering, Graduate School, Ajou University, Suwon, South Korea. His research interests include Bluetooth low energy (BLE) and

Internet of Things (IoT).

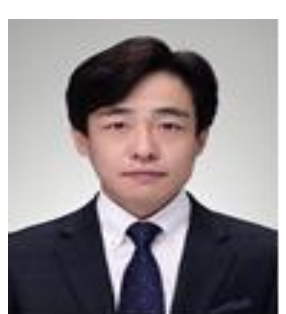

Seung-hun Shin received the B.S degree in information and computer engineering and M.S and Ph.D degrees in information and communication engineering from Ajou University, Suwon, South Korea, in 2000, 2002, and 2011, respectively. From September 2011 to February 2016, he was with the Department of Software Convergence Technology, Ajou University a s a lecture professor. Since March 2016, he has been with the Da-san University College, Ajou University, as an assistant professor. His research interests include software testing algorithms, network intrusion detection, and mobile multimedia networking.

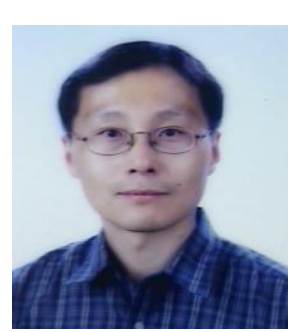

Byeong-hee Roh received the B.S degree in electronics engineering from Hanyang University, Seoul, South Korea, in 1987, and the M.S and Ph.D degrees in electrical engineering from the Korea Advanced Institute of Science and Technology, Daejeon, South Korea, in 1989 and 1998, respectively. From 1989 to 1994, he was with Telecommunication Networks Laboratory, Korea Telecom, as a Researcher. From February 1998 to March 2000, he worked with Samsung Electronics Co., Ltd., South Korea, as a senior engineer. Since March 2000, he has been with the Department of Software and Computer Engineering and the Department of Computer Engineering, Graduate School, Ajou University, Suwon, South Korea, where he is currently a professor. During 2005, he was a visiting associate professor with the Department of Computer Science, State University of New York, Stony Brook, NY, USA. During 2014, he was an adjunct researcher with the Agency for Defense Development, South Korea. His research interests include mobile multimedia networking, future Internet networks, Internet of Things platform and services, network security and cyber warfare, and military communications. 